\title{
Is the Use of Empirically Designed Custom Soft Contact Lenses a Good Option for Challenging Patients?
}

\author{
Frank Spors $^{1 *}$, Donald J. Egan ${ }^{1}$, Lance E. McNaughton ${ }^{1}$, Jie Shen ${ }^{1}$, Matthew J. Lampa ${ }^{2}$ \\ ${ }^{1}$ Western University of Health Sciences, College of Optometry, Pomona, USA; ${ }^{2}$ Pacific University, College of Optometry, Forest \\ Grove, USA. \\ Email: *fspors@westernu.edu
}

Received April 28 ${ }^{\text {th }}, 2013$; revised May 29 ${ }^{\text {th }}, 2013$; accepted June $15^{\text {th }}, 2013$

Copyright (C) 2013 Frank Spors et al. This is an open access article distributed under the Creative Commons Attribution License, which permits unrestricted use, distribution, and reproduction in any medium, provided the original work is properly cited.

\begin{abstract}
Purpose: Reasons for the lack of success for contact lens patients range from poor fit, to discomfort or to a less than optimum wearing schedule. This project was a preliminary investigation of customized soft toric contact lenses as an alternative for unsuccessful contact lens patients or potential drop-outs. Methods: Fifteen healthy non-compromised, previously unsuccessful contact lens wearing patients (13 women, 2 men) with ages from 21 to 33 years (25.1 \pm 2.7 years) were the subjects for this study. They were empirically fitted with 15 astigmatic and 5 spherical customized SpecialEyes soft contact lenses. Visual acuities, comfort, lens movement, and rotation were evaluated. Results: Patients fitted with customized SpecialEyes soft contact lenses had statistically significant better visual acuities than with Phoropter Manifest Refraction. The lenses used in this study centered well and moved no more than $1 \mathrm{~mm}$ upon each blink. Lens rotation was less than 10 degrees for all toric lenses. Patients gave very positive vision and comfort ratings. Conclusion: This preliminary study supports the application of a software program to empirically design and manufacture SpecialEyes custom soft lenses based upon corneal topographical parameters and subjective manifest refraction for those patients who either have challenging corneal parameters, high sphero-cylindrical prescriptions, or are currently dissatisfied with their contact lenses and are potential drop-outs.
\end{abstract}

Keywords: Soft Contact Lenses; Custom Contact Lenses; Toric Contact Lenses; Contact Lenses for Astigmatism; Empirical Contact Lens Fitting

\section{Introduction}

The goal of soft contact lens application is to combine excellent vision with overall lens comfort. In recent years the introduction of improved materials has led to an increase in the popularity of soft lenses, especially since frequent replacement options reduce the risk of microbial infections and the presence of lens deposits. As somewhat of a disadvantage however most frequent replacement lenses are only available in a narrow range of parameters. Indeed most lenses are available in just one diameter, one or two base curves, and in the case of astigmatic designs limited cylinder power and range of axis values exists. Although these limitations result in a simplified approach to lens selection, it may not be the best way of successfully prescribing contact lenses for patients who do not have average corneal shape values, refractive data, or visual demands.

"Corresponding author.
Up to $45 \%$ of the contact lens seeking population have astigmatism of $0.75 \mathrm{D}$ or greater [1]. However, toric soft contact lenses remain generally underutilized as a corrective strategy. A variety of soft toric contact lenses have been widely available for some years, but many practitioners are reluctant to incorporate them into their fitting routines or recommend them to wearers. This is partly due to a history of unpredictable lens orientation and difficulty in achieving a stable fit [1]. According to the International Survey of Contact Lens Prescribing, only $19 \%$ of prescribed daily wear soft contact lenses are toric in design [2].

The reasons for discontinuing contact lenses have been evaluated in a number of studies $[3,4]$. The most commonly cited reason in all of these studies is discomfort, which accounts for between $43 \%$ and $72 \%$ of the dropouts. Poor vision is another important factor which is due in part to product non-availability for high prescriptions, and practitioner misjudgment. Prime reasons for practi- 
tioner misjudgment are inappropriate lens fit and uncorrected astigmatism [5].

Lens reproducibility is regarded as another very important factor in the success of toric soft contact lenses, and poor reproducibility of frequent replacement lenses has been implicated in the underutilization of these lenses [6].

Selection of an initial soft contact lens base curve has traditionally been based on central corneal curvature, as measured by keratometry. The underlying assumption behind this is that corneas with steeper central radii have greater sagittal height and, therefore, require a lens of greater sagittal depth in the form of a steeper base curve to optimally fit the cornea. However, previous studies have shown that there is no strong correlation between keratometry readings and the best-fitting soft contact lens [7]. Accordingly soft toric contact lens fitting, when based on manifest refraction and keratometry alone, often presents unanticipated fitting and power errors upon initial lens dispensing [8].

Following the standard procedure of keratometry, empirical fitting success with soft toric contact lenses can be anticipated in spherical corneas, central astigmatism, or corneas with peripheral toricity matched with central toricity. Considering that high and irregular peripheral corneal toricity occurs in the majority of astigmatic patients, including empirical soft contact lens fitting based upon more corneal topographical parameters, sagittal height, and corneal diameter, may lead to increased fitting success, better visual acuity, and fewer drop-outs [8-10]. Incorporation of all of these parameters requires customized soft contact lenses with varying base curves, diameters, sagittal heights, and power values [11,12].

The purpose of this project was to evaluate SpecialEyes customized soft toric contact lenses as an alternative for unsuccessful contact lens patients or potential drop-outs.

\section{Methods}

\subsection{Subjects and Inclusion Criteria}

Fifteen healthy, non-compromised, previously unsuccessful contact lens wearing patients (13 women, 2 men) with ages ranging from $21-33$ years $(25.1 \pm 2.7$ years $)$ were the subjects for this study. The reasons for previous drop-outs were unsatisfactory visual acuity and/or reduced comfort with conventional and disposable soft contact lenses. Refractive errors were not limited, but fell within the range of $-8.00 \mathrm{D}$ to $+3.75 \mathrm{D}$ sphere, and up to $-3.75 \mathrm{D}$ of spectacle cylinder. Expressed in rectangular Fourier optics terms, the mean spherical equivalent was $M=-4.36 \mathrm{D}(\mathrm{SD} 2.66 \mathrm{D})$, and the astigmatic vector components were $J_{0}=0.09 \mathrm{D}(\mathrm{SD} 0.74 \mathrm{D})$, and $J_{45}=$ $0.04 \mathrm{D}$ (SD $0.62 \mathrm{D}$ ). None of the participants suffered from any eye disease or injury, nor were they taking any medication or supplements. This research followed the tenets of the Declaration of Helsinki, and informed consent was obtained from all participants. All measurements were conducted at the Health Education Center at Western University of Health Sciences, College of Optometry.

\subsection{Measurements and Fitting Modalities}

Corneal topography data were obtained using the Medmont E 300 ${ }^{\mathrm{TM}}$ Corneal Topography System (Medmont Pty Ltd, Nudawading, VIC, Australia). Data of interest included tangential curvature, elevation topographical maps, simulated keratometry values, and corneal diameters, as depicted in Figure 1. In addition, the patients' maximum plus manifest non-cycloplegic Phoropterrefractions were measured to determine lens power values. Each patient's best corrected high contrast visual acuities were obtained using ETDRS letters provided by the M\&S Smart System (M\&S Technologies, Inc., Niles, IL, USA) and recorded in logarithmically-stepped Snellen foot notation. The examination room illumination was 100 lux.

Thirty eyes were fitted with custom hydrogel contact lenses, empirically designed and manufactured by SpecialEyes (Bradenton, FL, USA) based upon the submitted topographical and refractive data.

All contact lenses were initially evaluated and subsequently followed-up to determine if this approach in lens design represents a successful alternative to generic lens designs which account for the standard office stock available and dispensed by most eye care practitioners.

Corneal anatomical features and refraction data determined the lens parameters designed by SpecialEyes' proprietary software. From the available data the following contact lens parameters were calculated:

- Diameter

- Base curve

- Sagittal height

- Power

\subsection{Contact Lenses and Lens Material}

All lenses were used as daily wear lenses. Each lens was a customized lathe-cut design manufactured by SpecialEyes from the p-GMA/HEMA hioxifilcon D material with a water content of $54 \%$, and a Dk value of $23 \times 10^{-11}$ $(\mathrm{cm} / \mathrm{sec})\left(\mathrm{mLO}_{2} / \mathrm{mL} / \mathrm{mm} \mathrm{Hg}\right)$. All lenses were designed for a three month replacement modality. Table 1 lists the range of contact lens parameters used in this study.

Twenty-five lenses were astigmatic, and five lenses were spherical. All patients were fit with at least one astigmatic lens. Eighteen eyes had with-the-rule astigmatism, three eyes had against-the-rule astigmatism, and 

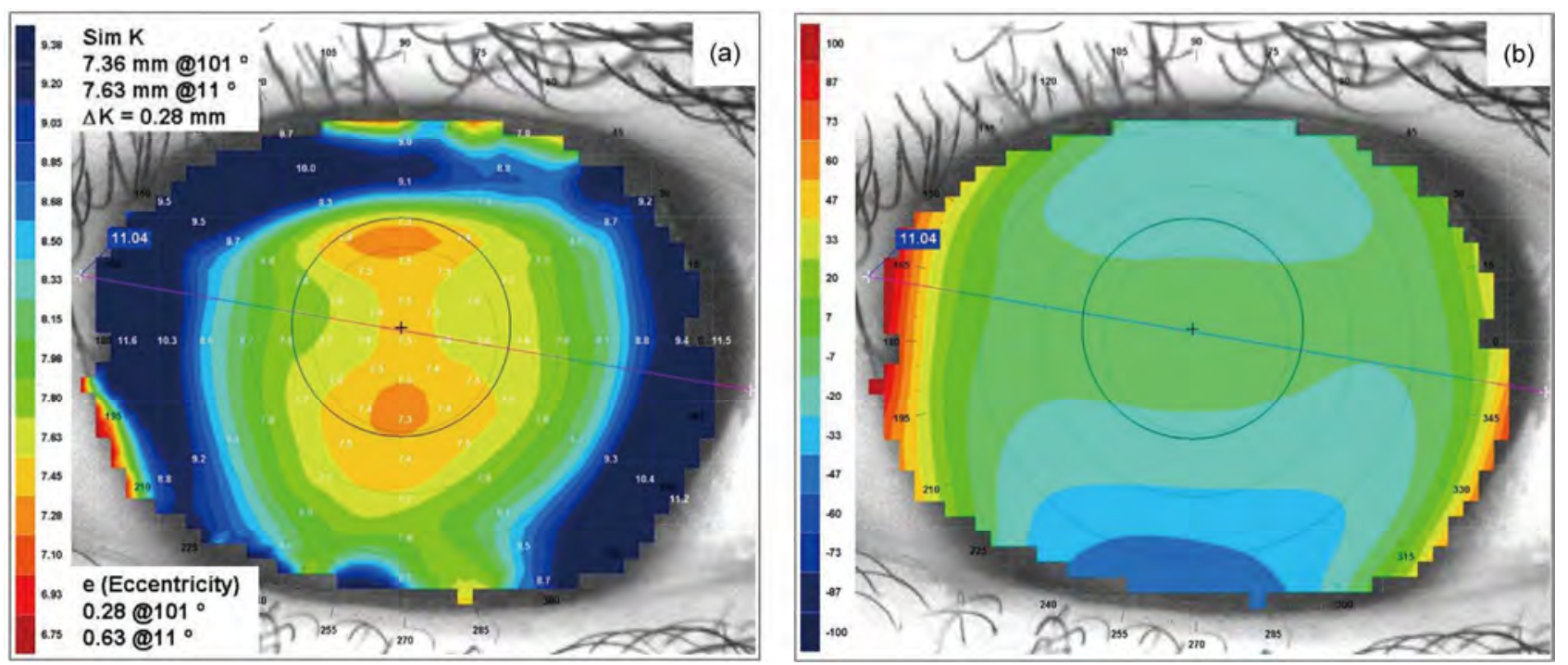

Figure 1. Corneal topographical maps: (a) Tangential curvature; (b) Elevation.

Table 1. Range of SpecialEyes contact lens parameters used in this study.

\begin{tabular}{cccc}
\hline Parameter & Range & Mean & SD \\
\hline Base curve & $7.70 \mathrm{~mm}-9.00 \mathrm{~mm}$ & $8.42 \mathrm{~mm}$ & $0.40 \mathrm{~mm}$ \\
Diameter & $13.80 \mathrm{~mm}-15.20 \mathrm{~mm}$ & $14.54 \mathrm{~mm}$ & $0.39 \mathrm{~mm}$ \\
Sphere power & $-8.00 \mathrm{D}-+3.75 \mathrm{D}$ & $-3.69 \mathrm{D}$ & $2.51 \mathrm{D}$ \\
Cylinder power & $-3.25 \mathrm{D}--0.50 \mathrm{D}$ & $-1.47 \mathrm{D}$ & $0.81 \mathrm{D}$ \\
Central thickness & $99.43 \mu \mathrm{m}-284.66 \mu \mathrm{m}$ & $120.49 \mu \mathrm{m}$ & $38.91 \mu \mathrm{m}$ \\
Sagittal height & $3129.87 \mu \mathrm{m}-4470.68 \mu \mathrm{m}$ & $3784.10 \mu \mathrm{m}$ & $278.73 \mu \mathrm{m}$ \\
\hline
\end{tabular}

four eyes had oblique astigmatism.

SpecialEyes employs a prism ballast stabilization design for back surface toric lenses, which is depicted in Figure 2. In general, this design is associated with a higher visual acuity when compared to alternative stabilization mechanisms [13]. To assure optimum optical quality each of the manufactured lenses was evaluatedprior to delivery with a Nimo TR1504 contact lens power mapper and wavefront analyzer (Lambda-X S.A., Nivelles, Belgium) via the Phase-Shifting Schlieren transmission method. Sample images of these measurements are displayed in Figure 3.

\subsection{Fitting Evaluation}

Lenses were allowed to settle for fifteen minutes, followed by evaluation of centration, movement, and in the case of astigmatic lenses, lens rotation. Fitting was evaluated with a Haag Streit BQ 900 slit lamp utilizing the IM 900 digital imaging system and EyeCap Version 6.2.0 software (Haag Streit AG, Koeniz, Switzerland).

In observance of Snyder's Rule the maximum rotation permitted for a successful fit was no more than ten degrees [14]. High contrast visual acuity was measured under the initial conditions, and all patients rated vision and comfort with their lenses on a scale from 1 (not satisfied) to 5 (excellent).

Fitting success was determined as indicated by the following parameters:

- Visual Acuity: with SpecialEyes contact lenses equal to or better than Phoropter Manifest Refraction.

- Subjective Vision Rating: at least 4 (very satisfied) for the majority of lenses, and no value of 1 (not satisfied) for any lens.

- Subjective Comfort Rating: at least 4 (very satisfied) for the majority of lenses, and no value of 1 (not satisfied) for any lens.

- Lens Rotation: less than 10 degrees for astigmatic lenses.

- Lens Decentration: between $0.1 \mathrm{~mm}$ and $1.0 \mathrm{~mm}$ postblink movement, and inter-blink stability.

\subsection{Statistical Analysis}

A Wilcoxon matched-pairs signed rank test was conducted for thirty eyes with Prism6 (GraphPad Software Inc., La Jolla, USA) after not passing the KolmogorovSmirnov normality test with Dallal and Wilkinson ap- 

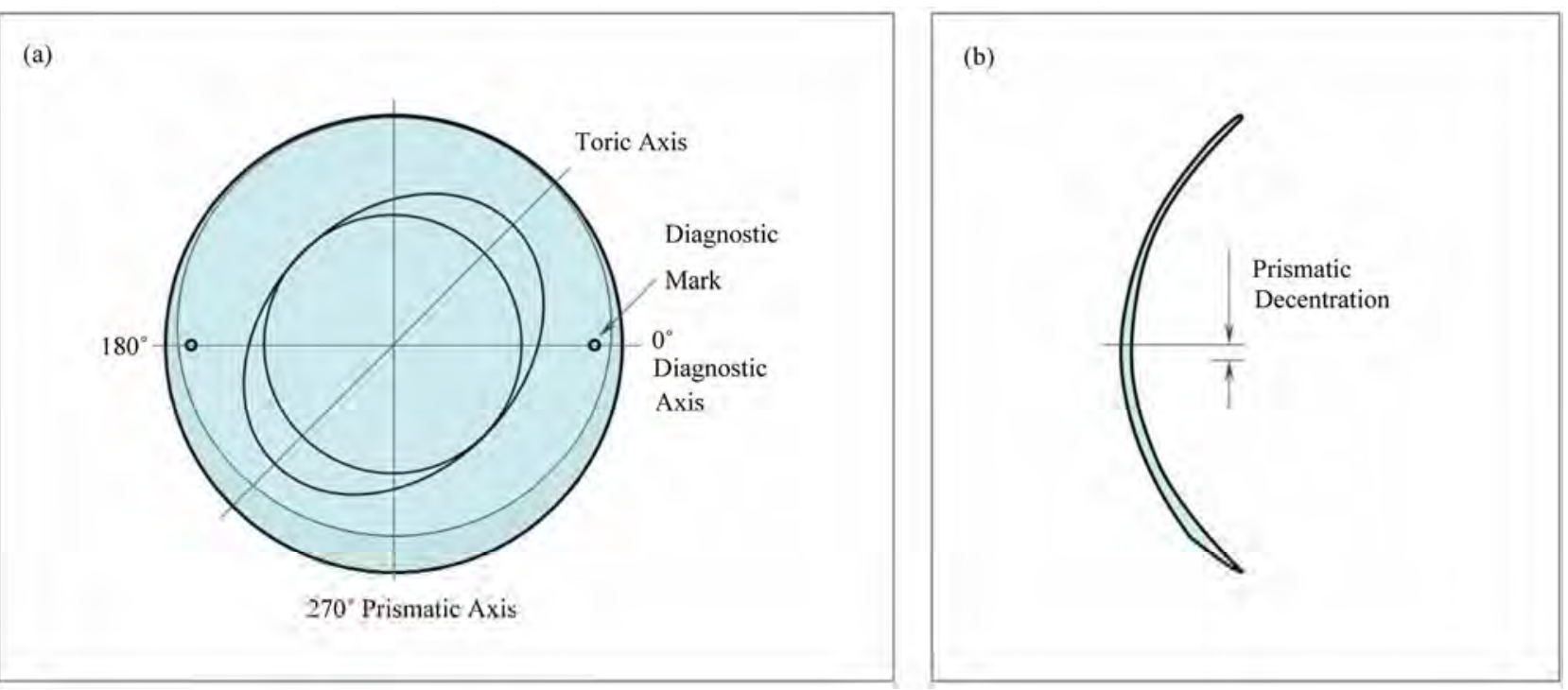

Figure 2. SpecialEyes toric soft contact lens, prism ballast stabilization example: (a) Front view showing markings, toric optic zone, and prism distribution; (b) Cross-sectional view illustrating prism distribution.
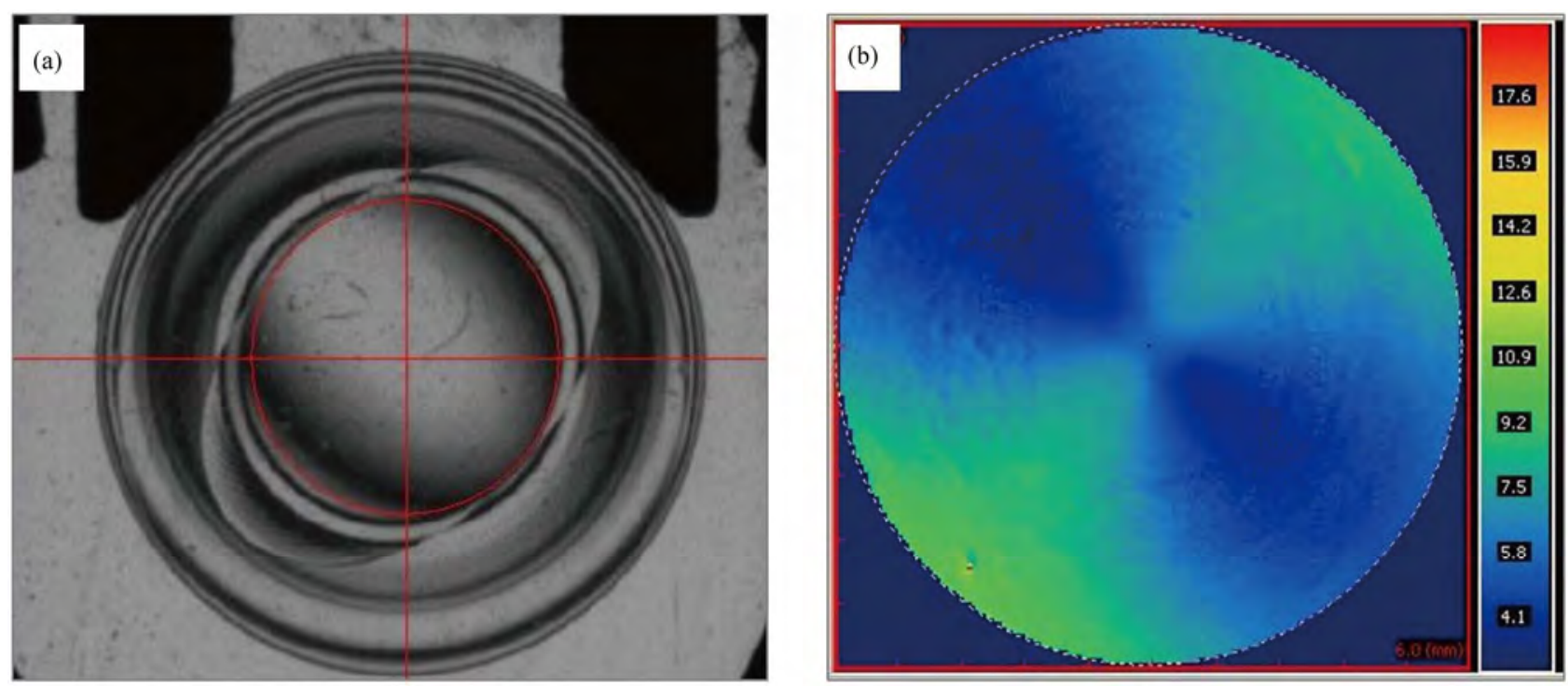

Figure 3. Nimo TR 1504 images of a SpecialEyes soft toric contact lens: (a) Phase-shifting Schlieren image; (b) Wavefront analysis of the central $6 \mathrm{~mm}$ optical zone of the same lens, (Images courtesy SpecialEyes).

proximation to Lilliefors' method ( $P<0.0001)$ [15].

The mean visual acuity values achieved with the Phoropter Manifest Refraction and with the SpecialEyes lenses were compared. For statistical purposes, a $P$ value lower than 0.05 was considered statistically significant.

\section{Results}

The analysis of mean visual acuity displayed in Figure 4 and Table 2 illustrates that patients fit with customized SpecialEyes soft contact lenses had a statistically significant better visual acuities than with Phoropter Manifest Refraction. All patients evaluated the contact lenses with regards to vision and comfort and gave ratings of at least 4 (very satisfied) for the majority of lenses, whereas no one reported a value of 1 (not satisfied) for any lens, as shown in Figure 5. Judgments of lens and axis rotation as well as movement upon blink are depicted in Figure 6. The lenses used in this study centered well and moved smoothly over the patients' corneas and conjunctivas. Figure 7 shows two representative slit lamp biomicroscopy images.

\section{Discussion}

The results of this preliminary study support the application of a software program to empirically design and 


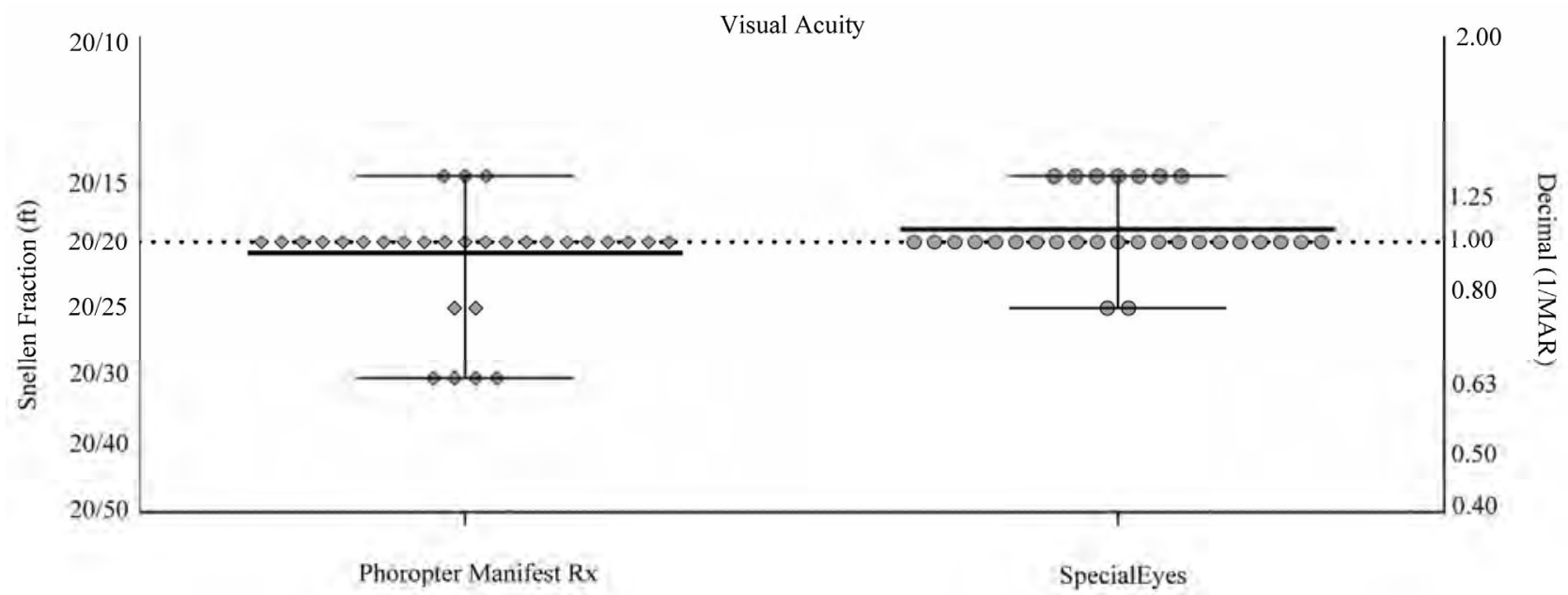

Figure 4. Visual acuities achieved with Phoropter Manifest Refraction and SpecialEyes lenses.

Table 2. Statistical analysis of differences in visual acuities achieved with Phoropter Manifest Refraction versus SpecialEyes lenses.

\begin{tabular}{|c|c|c|c|}
\hline \multicolumn{4}{|c|}{ Wilcoxon matched-pairs signed rank test } \\
\hline Treatment modality & Phoropter Manifest Rx & & SpecialEyes \\
\hline Number of values & 30 & & 30 \\
\hline Mean & 0.9623 & & 1.045 \\
\hline Std. Deviation & 0.1632 & & 0.1255 \\
\hline Std. Error of Mean & 0.02980 & & 0.02291 \\
\hline Lower 95\% CI of mean & 0.9014 & & 0.9981 \\
\hline Upper 95\% CI of mean & 1.023 & & 1.092 \\
\hline$P$ value & \multicolumn{3}{|c|}{0.0208} \\
\hline Significantly different? $(P<0.05)$ & \multicolumn{3}{|c|}{ Yes } \\
\hline One- or two-tailed $P$ value? & \multicolumn{3}{|c|}{ Two-tailed } \\
\hline Sum of positive, negative ranks & \multicolumn{3}{|c|}{$88.50,-16.50$} \\
\hline Sum of signed ranks (W) & \multicolumn{3}{|c|}{72.00} \\
\hline
\end{tabular}
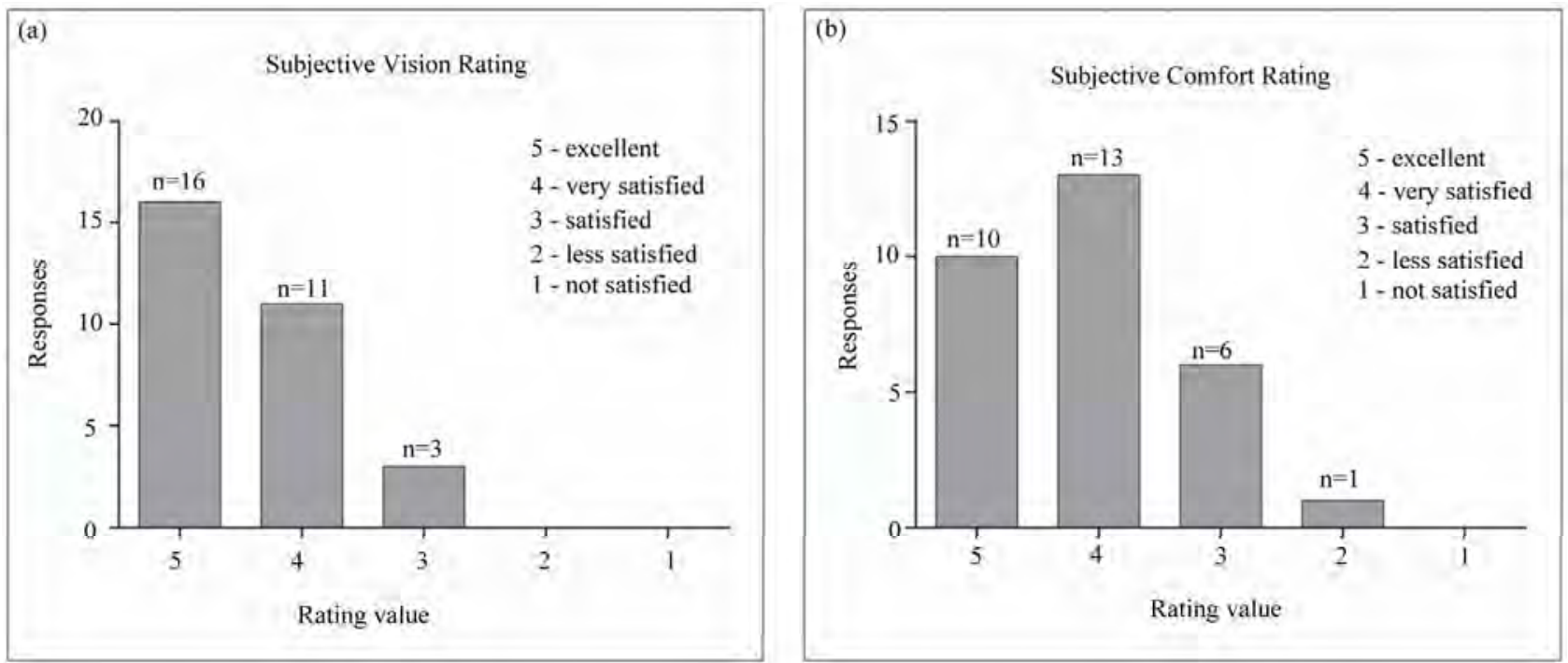

Figure 5. Subjective ratings for SpecialEyes soft contact lenses: (a) Vision; (b) Comfort. 

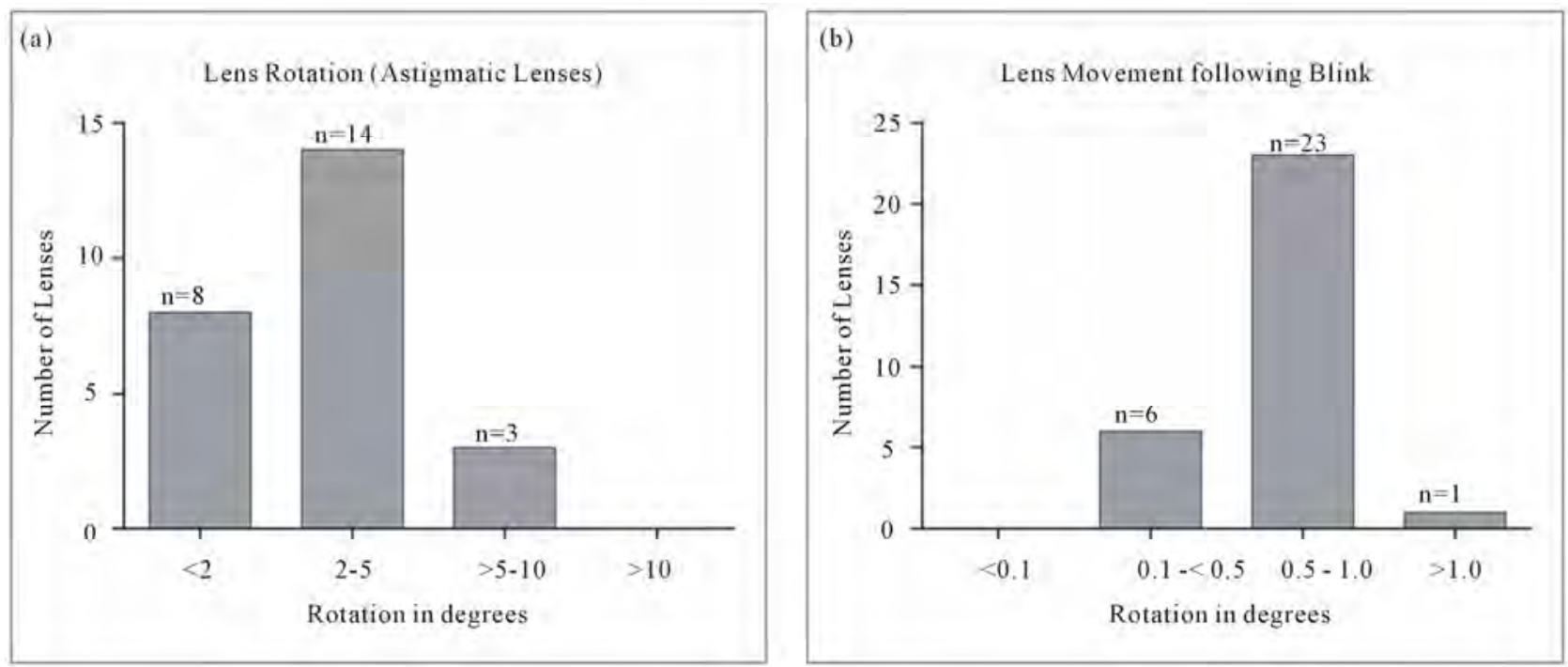

Figure 6. SpecialEyes lenses observed by biomicroscopy: (a) Rotation; (b) Movement.
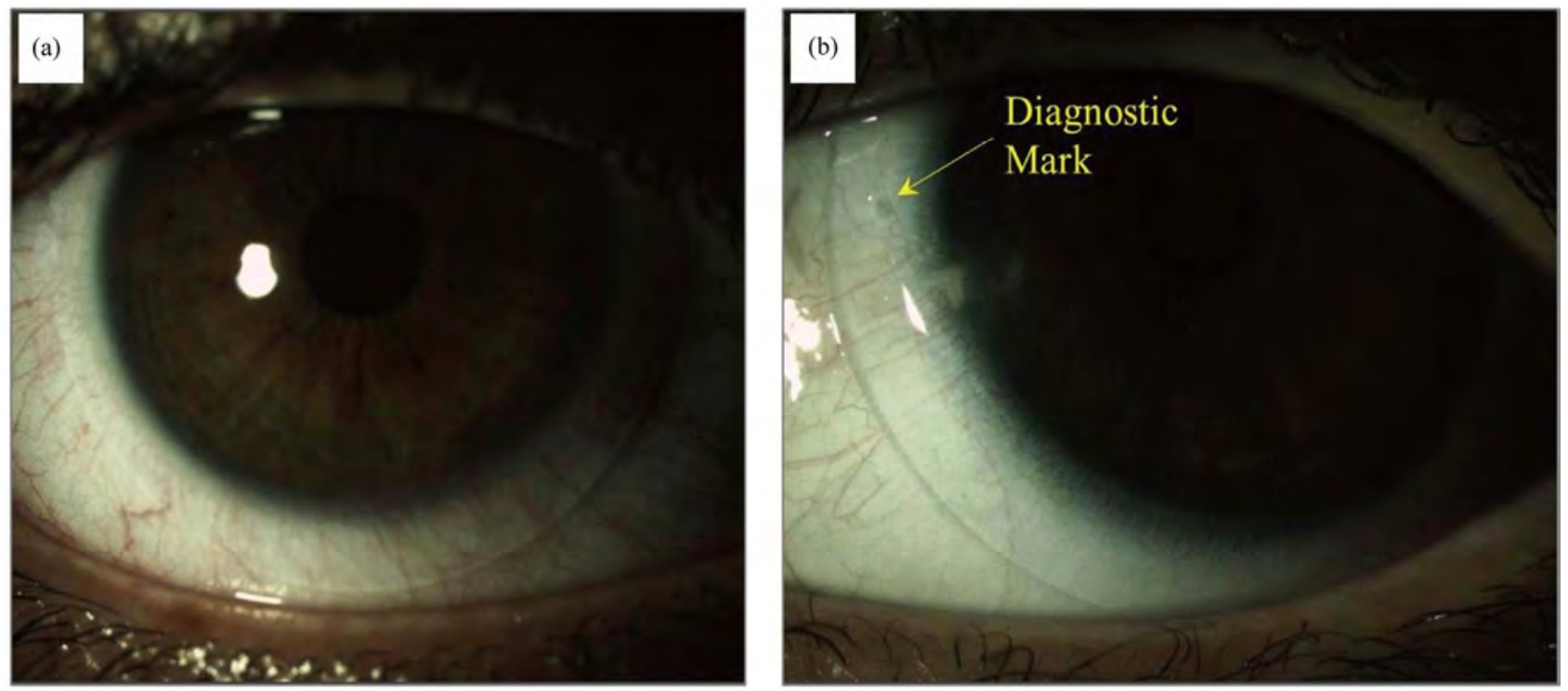

Figure 7. Biomicroscopy images of SpecialEyes custom soft lenses: (a) Spherical lens; (b) Astigmatic lens.

manufacture SpecialEyes custom soft lenses based upon corneal topographical parameters and subjective manifest refraction data for those patients who either have challenging corneal parameters, high sphero-cylindrical prescriptions, or are currently dissatisfied with their contact lenses and are potential drop-outs. Customized lens parameters, a robust stabilization mechanism for astigmatic lenses, a comfortable hydrophilic material, in vivo parameter stability, and high reproducibility are shown here to be important elements for successful fitting soft contact lenses in challenging patients.

Lenses made from the hioxifilcon material family, as used in this study, are known to remain saturated and dimensionally stable on the eye resulting in excellent visual acuity, comfort, as well as alleviation of symptoms and ocular surface staining associated with contact lens related dryness [16].

The patients in our study reported good comfort and vision with customized SpecialEyes soft contact lenses. With these lenses the patients achieved statistically significant improvements in visual acuities when compared with Phoropter Manifest Refraction, although this difference was not clinically significant, since the visual acuity improved less than one line. Possible explanations for the increase in visual acuity with lenses when compared to the best corrected manifest phoropter refraction may be larger retinal image sizes in myopic patients, and the reduction of some corneal higher order aberrations, 
e.g. coma, due to the thickness of the hioxifilcon D contact lens material [13,17].

\section{Conclusions}

The results of this study suggest that empirically designed custom fit soft contact lenses:

- Fit most refractive parameter combinations and corneal profiles;

- Allow fast and easy correction of high astigmatic refractive errors;

- Provide good patient comfort;

- Reduce chair-time;

- Increase the first pair fit success rate.

The virtually unlimited range of parameters with these lenses assures availability of the optimal lens for each patient's condition, and may have a positive impact on patient compliance and loyalty. A rigorous cleaning and disinfecting regimen in combination with planned lens replacement is necessary to maintain optical quality as well as hydrophilicity, and to decrease the accumulation of long term deposits on the contact lens material. It appears that a 3-month replacement cycle is appropriate for these lathe-cut manufactured custom soft lenses.

Additional research with a larger sample of normal as well as compromised corneas, different lens types and materials is planned for future studies.

\section{Acknowledgements}

We thank SpecialEyes, especially Ms. Lindsay McCorkle, for empirically designing and providing the customized contact lenses used in this study.

\section{REFERENCES}

[1] J. Tan, E. Papas, N. Carnt, et al., "Performance Standards for Toric Soft Contact Lenses,” Optometry \& Vision Science, Vol. 84, No. 5, 2007, pp. 422-428. doi:10.1097/OPX.0b013e318059063b

[2] N. Efron, P. B. Morgan and C. A. Woods, "International Survey of Contact Lens Prescribing for Extended Wear," Optometry \& Vision Science, Vol. 89, No. 2, 2012, pp. 122-129. doi:10.1097/OPX.0b013e31823ee077

[3] N. Pritchard, D. Fonn and D. Brazeau, "Discontinuation of Contact Lens Wear: A Survey,” International Contact Lens Clinic, Vol. 26, No. 6, 1999, pp. 157-162. doi:10.1016/S0892-8967(01)00040-2

[4] J. L. Schlanger, "A Study of Contact Lens Failures," Journal of the American Optometric Association, Vol. 64, No. 3, 1993, pp. 220-224.
[5] G. Young, "Why One Million Contact Lens Wearers Dropped out," Contact Lens \& Anterior Eye, Vol. 27, No. 2, 2004, pp. 83-85.

[6] G. Young, S. Coleman and S. Hickson-Curran, "Clinical Evaluation of Toric Soft Lens Reproducibility,” Optometry \& Vision Science, Vol. 79, No. 5, 2002, pp. 321-328. doi:10.1097/00006324-200205000-00012

[7] L. A. Hall, G. Young, J. S. Wolffsohn and C. Riley, “The Influence of Corneoscleral Topography on Soft Contact Lens Fit,” Investigative Ophthalmology \& Visual Science, Vol. 52, No. 9, 2011, pp. 6801-6806. doi:10.1167/iovs.11-7177

[8] L. B. Szczotka, C. Roberts, E. E. Herderick and A. Mahmoud, "Quantitative Descriptors of Corneal Topography that Influence Soft Toric Contact Lens Fitting,” Cornea, Vol. 21, No. 3, 2002, pp. 249-255. doi:10.1097/00003226-200204000-00003

[9] T. Reddy, L. B. Szczotka and C. Roberts, "Peripheral Corneal Contour Measured by Topography Influences Soft Toric Contact Lens Fitting Success,” CLAO Journal, Vol. 26, No. 4, 2000, pp. 180-185.

[10] P. J. Caroline and M. P. Andre, "The Effect of Corneal Diameter on Soft Lens Fitting, Part 1," Contact Lens Spectrum, 1 April 2002.

[11] P. J. Caroline and M. P. Andre, "The Effect of Corneal Diameter on Soft Lens Fitting, Part 2,” Contact Lens Spectrum, 1 May 2002.

[12] R. L. Davis and P. D. Becherer, “Techniques for Improved Soft Lens Fitting," Contact Lens Spectrum, $1 \mathrm{Au}-$ gust 2005.

[13] D. A. Berntsen, M. M. Merchea, K. Richdale, C. J. Mack and J. T. Barr, "Higher-Order Aberrations When Wearing Sphere and Toric Soft Contact Lenses,” Optometry \& Vision Science, Vol. 86, No. 2, 2009, pp. 115-122. doi:10.1097/OPX.0b013e318194e951

[14] C. Snyder, "Evaluation of 'High-Cylinder' Toric Soft Contact Lenses,” International Contact Lens Clinic, Vol. 24, No. 5, 1997, pp. 160-165. doi:10.1016/S0892-8967(97)00057-6

[15] G. E. Dallal and L. Wilkinson, “An Analytic Approximation to the Distribution of Lilliefors Test Statistic for Normality," The American Statistician, Vol. 40, No. 4, 1986, pp. 294-296.

[16] C. Riley, R. L. Chalmers and N. Pence, "The Impact of Lens Choice in the Relief of Contact Lens Related Symptoms and Ocular Surface Findings," Contact Lens \& Anterior Eye, Vol. 28, No. 1, 2005, pp. 13-19. doi:10.1016/j.clae.2004.09.002

[17] J. Shen and L. N. Thibos, "Peripheral Aberrations and Image Quality for Contact Lens Correction,” Optometry \& Vision Science, Vol. 88, No. 10, 2011, pp. 1196-1205. doi:10.1097/OPX.0b013e3182279fd5 